\title{
Interpolation Theory on Sobolev Spaces over the Abstract Wiener Space
}

\author{
Takuya SOBUKAWA \\ Keio University \\ (Communicated by Y. Ito)
}

\begin{abstract}
In recent years, the theory of Sobolev spaces over the abstract Wiener space was constructed in the study of stochastic differential equations $([9,11,15])$. In the present paper, we prove an interpolation theorem on Sobolev spaces over the abstract Wiener space.
\end{abstract}

\section{§ 1. Introduction.}

The following interpolation theorem on Sobolev spaces over the Euclidean space $\boldsymbol{R}^{n}$ with the ordinary Lebesgue measure

$$
W^{s, p}\left(\boldsymbol{R}^{n}\right)=\left\{f \in L_{1 \mathrm{oc}}^{1}\left(\boldsymbol{R}^{n}\right) \mid\left\|(I-\Delta)^{s} f\right\|_{p}<\infty\right\}
$$

( $s \in R, 1<p<\infty$, and $\Delta$ is the Laplace operator) is well-known:

THEOREM A (cf. Theorem 6.4.5 of [1]). If $s_{0}, s_{1} \in \boldsymbol{R}$ and $1<p_{0}, p_{1}<\infty$,

$$
\left[W^{s_{0}, p_{0}}, W^{s_{1}, p_{1}}\right]_{\theta}=W^{s, p}
$$

where

$$
s=(1-\theta) s_{0}+\theta s_{1}, \quad \frac{1}{p}=\frac{1-\theta}{p_{0}}+\frac{\theta}{p_{1}}, \quad 0<\theta<1 .
$$

Here, $[X, Y]_{\theta}$ is an interpolation space between the Banach spaces $X, Y$ (about this notation, see [1] Chapter 4).

Usually, Theorem A is próved by using the "Littlewood-Paley theory" as follows.

Lemma B. Let $1<p<\infty$ and $s \in \boldsymbol{R}$. Then,

$$
W^{s, p}(\boldsymbol{R}) \sim L^{p}\left(l_{2}^{s}\right)
$$

where $L^{p}\left(l_{2}^{s}\right)$ is the space of $l_{2}^{s}$-valued $L^{p}$-functions and 


$$
l_{2}^{s}=\left\{\left(a_{n}\right)_{n=-\infty}^{\infty} \mid a_{n} \in C \text { for any } n \text { and }\left\|\left(a_{n}\right)\right\|_{l_{2}^{s}}=\left[\sum_{n=-\infty}^{\infty}\left(2^{s n} a_{n}\right)^{2}\right]^{1 / 2}<\infty\right\}
$$

(see Chapter 7 of [2] and Theorem 6.4.3 of [1]).

In this paper, we shall consider the Sobolev spaces over the abstract Wiener space $(B, H, \mu)$, where $B$ is a separable real Banach space with the norm $\|\cdot\|_{B}, H$ is a separable real Hilbert space with the inner product $\langle\cdot, \cdot\rangle_{H}$ and the norm $|\cdot|_{H}=\sqrt{\langle\cdot, \cdot\rangle_{H}}$, which is densely and continuously imbedded into $B$, and $\mu$ is a normal mean zero Gaussian measure on $B$ i.e. a Borel probability measure on $B$ which satisfies

$$
\int_{B} e^{i \varphi(w)} d \mu(w)=\exp \left(-\frac{1}{2}|\varphi|_{H}^{2}\right) \quad \text { for every } \varphi \in B^{*} .
$$

As is known, one of the most important examples of the abstract Wiener space is the Gaussian-Euclidean space, the case $B=H=\boldsymbol{R}^{n}$, Euclidean space, and $\mu$ is the Gaussian measure

$$
d \mu(x)=\frac{1}{(2 \pi)^{n / 2}} \exp \left(-\frac{1}{2}|x|^{2}\right) d x
$$

where $d x$ is the Lebesgue measure on $\boldsymbol{R}^{n}$.

However, the Gaussian-version of Lemma B is unknown. So, in this paper, we shall show the similar theory over the abstract Wiener space $(B, H, \mu)$ straightforwards. In $\S 2$, we shall state some definitions and basic theorems. In $\S 3$, we shall show the $L^{p}$-boundedness of "Bessel potential" operators $(I-L)^{i y}$, where $y \in R$ and $L$ is the Ornstein-Uhlenbeck operator which plays a role of Laplacian operator over the abstract Wiener spaces. And in $\S 4$, we shall show an interpolation theorem on the Sobolev spaces over the abstract Wiener space $(B, H, \mu)$ by using the results of $\S 3$.

Here the author wishes to express his thanks to Professor S. Koizumi and Professor S. Ishikawa for their valuable advices and encouragement, and to the referee for his valuable advice.

\section{§ 2. Basic notations.}

Let $(B, H, \mu)$ be the abstract Wiener space. We shall state several definitions.

DEFINITION 2.1. Let $\left\{\phi_{n}\right\}_{n=1}^{\infty} \subset B^{*}$ be complete orthonormal system in $H$, and let $p$ be a polynomial of several variables with complex coefficients. Then a function on $B$, 


$$
f(w)=p\left(\varphi_{1}(w), \varphi_{2}(w), \cdots, \varphi_{n}(w)\right),
$$

is said to be a polynomial on $B$. We call the degree of $p$ the one of $f$ and a family of all polynomials is denoted by $\boldsymbol{P}$. It is known that $\boldsymbol{P}$ is a dense subset of $L^{p}(B, \mu)(1 \leqq p<\infty)$ (see [5]).

Definition 2.2 (The Ornstein-Uhlenbeck semigroup). For $t \geqq 0$, we define an operator $T_{t}$ by

$$
T_{t} f(w)=\int_{B} f\left(e^{-t} w+\sqrt{1-e^{-2 t}} v\right) d \mu(v) \quad \text { for every } f \in P .
$$

The family $\left\{T_{t}\right\}_{t \geq 0}$ is a $C_{0}$-contraction symmetric semigroup in $L^{p}(B, \mu)$ for every $1 \leqq p<\infty$ (cf. [6]). We call it the Ornstein-Uhlenbeck semigroup, and the infinitesimal generator $L$ of it is called the Ornstein-Uhlenbeck operator. As is known, $L^{2}(B, \mu)$ is decomposed into orthogonal direct sum;

$$
L^{2}(B, \mu)=\bigoplus_{n=0}^{\infty} C_{n},
$$

called Wiener-Itô decomposition, where $C_{0}=\{$ the set of all constant functions\} and

$C_{n}=\left\{L^{2}\right.$-closure of the set of all polynomials of degree $\left.n\right\} \ominus\left(C_{0} \oplus \cdots \oplus C_{n-1}\right)$ for $n \geqq 1$. Here we define that $J_{n}$ is the orthogonal projection from $L^{2}(B, \mu)$ to $C_{n}(n=0,1,2, \cdots)$. From the property of $C_{n}$, we have

$$
L f=\sum_{n=0}^{\infty}(-n) J_{n} f \quad(f \in \boldsymbol{P})
$$

and hence

$$
T_{t} f=\sum_{n=0}^{\infty} e^{-n t} J_{n} f \quad(f \in \boldsymbol{P}) .
$$

We shall consider multiplier operator $M_{m}: \boldsymbol{P} \rightarrow \boldsymbol{P}$ given by

$$
M_{m} f=\sum_{n=0}^{\infty} m(n) J_{n} f \quad \text { for every } f \in \boldsymbol{P}
$$

where $m(n)$ is a complex sequence. The $L^{p}$-boundedness of such $M_{m}$ is an interesting problem. P. A. Meyer and I. Shigekawa showed it in a certain case.

Theorem 2.1 (P. A. Meyer-I. Shigekawa [11], [13]). If $m(n)$ is a real sequence and there exist a function $h(\cdot)$ which is analytic on some 
neighbourhood of the origin and a positive constant $\alpha$ such that $m(n)=$ $h\left(n^{-\alpha}\right)$, then $M_{m}$ can uniquely extend to a bounded linear operator on $L^{p}$ for each $1<p<\infty$.

From (2.3), it is natural to define

$$
(I-L)^{\varepsilon} f=\sum_{n=0}^{\infty}(1+n)^{2} J_{n} f \quad \text { for every } f \in P, s \in C,
$$

and to call it "Bessel potential" operator. Now we define the Sobolev spaces over the abstract Wiener space.

Definition 2.3. For $s \in R, 1<p<\infty$, let $W^{s, p}$ be the completion of $P$ by the norm $\|\cdot\|_{s, p}$, where

$$
\|f\|_{s, p}=\left\|(I-L)^{s / 2} f\right\|_{p} \quad \text { for every } f \in P \text {. }
$$

We shall state a property of $W^{s, p}$ (see [13]).

Proposition 2.2. The dual space $W^{s, p}$ is $W^{-s, p^{\prime}}$ where $1 / p+1 / p^{\prime}=1$ $(1<p<\infty, s \in \boldsymbol{R})$.

§3. The Marcinkiewicz-Stein multiplier theorem.

E. M. Stein proved the compact Lie group version of the "Marcinkiewicz multiplier theorem" by using the Littlewood-Paley theory [12]. He also said that it can be extended to the general case, but the proof of it is informal. In this section, we prove the abstract Wiener space version of "Marcinkiewicz theorem" rigorously and prove, as a corollary, the $L^{p}$-boundedness of the "Bessel potential" operator $(I-L)^{i y}$, where $y \in \boldsymbol{R}$.

Let $0<\beta \leqq 1$ and we define the operator $\boldsymbol{T}_{t}^{(\beta)}: \boldsymbol{P} \rightarrow \boldsymbol{P}$ by

$$
T_{t}^{(\beta)} f=\sum_{n=0}^{\infty} e^{-n^{\beta} t} J_{n} f \quad \mu \text {-a.e. for every } f \in P .
$$

It is known that $T_{t}^{(\beta)}$ is a $C_{0}$-semigroup and has four more properties as follows.

1. (Contractivity)

For $1 \leqq p \leqq \infty$ and for every $f \in L^{p}(B, \mu)$,

$$
\left\|T_{t}^{(\beta)} f\right\|_{p} \leqq\|f\|_{p}
$$

2. (Self-adjointness)

For every $f, g \in L^{2}(B, \mu)$,

$$
\int_{B}\left(T_{t}^{(\beta)} f(w)\right) g(w) d \mu(w)=\int_{B} f(w)\left(T_{t}^{(\beta)} g(w)\right) d \mu(w) .
$$


3. (Positivity)

If $f(w) \geqq 0$ for almost all $w \in B, T_{t}^{(\beta)} f(w) \geqq 0$ for almost all $w \in B$.

4. (Conservativity)

$$
T_{t}^{(\beta)} 1=1
$$

In general the $C_{0}$-contraction semigroup which satisfies these four properties is called the symmetric diffusion semigroup. For such a semigroup, the Littlewood-Paley theory was constructed.

TheOREM 3.1 (E. M. Stein [12]). Let $0<\beta \leqq 1$. For $f \in P$, we define

$$
g_{k, \beta}(f)(w)=\left[\int_{0}^{\infty} t^{2 k-1}\left|\frac{\partial^{k}}{\partial t^{k}} T_{t}^{(\beta)} f(w)\right|^{2} d t\right]^{1 / 2} \quad(k=1,2, \cdots) .
$$

Then, for $1<p<\infty$, there exist some constants $a_{p, k, \beta}, A_{p, k, \beta}$ which depend only on $p, k, \beta$ such that

$$
a_{p, k, \beta}\left\|\left(I-J_{0}\right) f\right\|_{p} \leqq\left\|g_{k, \beta}(f)\right\| \leqq A_{p, k, \beta}\|f\|_{p} \text {. }
$$

Now we consider the Marcinkiewicz-Stein multiplier theorem.

DEFinition 3.1. Let $a(\cdot)$ be a bounded complex function on $[0, \infty)$. Then we define the complex function $m(\cdot)$ as follows;

$$
m(\lambda)= \begin{cases}0, & \text { for } \lambda=0 \\ \lambda \int_{0}^{\infty} e^{-\lambda s} \alpha(s) d s, & \text { for } \lambda>0,\end{cases}
$$

and call it a function of Laplace transform type.

THEOREM 3.2. Let $m(\cdot)$ be a function of Laplace transform type and $0<\beta \leqq 1$. Then the multiplier operator

$$
M_{m} f(w)=\sum_{n=0}^{\infty} m\left(n^{\beta}\right) J_{n} f(w), \quad f \in \boldsymbol{P}
$$

is $L^{p}$ bounded $(1<p<\infty)$, i.e.,

$$
\left\|M_{m} f\right\|_{p} \leqq A_{p}\|f\|_{p} \quad \text { for every } f \in P
$$

where the positive constant $A_{p}$ depends only upon $p$.

Proof. Let us take $f \in P$ and fix it, and let us put

$$
F(w)=-\int_{0}^{\infty} \frac{\partial}{\partial t} T_{t}^{(\beta)} f(w) \cdot a(t) d t
$$

Then $F(w)=M_{m} f(w)$. In fact, since $J_{n} f=0$ for all but finite $n$, we have 


$$
\begin{aligned}
\int_{0}^{\infty} \frac{\partial}{\partial t} T_{t}^{(\beta)} f(w) a(t) d t & =\sum_{n=0}^{\infty} \int_{0}^{\infty}-n^{\beta} e^{-n^{\beta} t} J_{n} f(w) a(t) d t \\
& =\sum_{n=0}^{\infty}-m\left(n^{\beta}\right) J_{n} f(w) .
\end{aligned}
$$

Applying the operator $T_{t_{1}}^{(\beta)}$ to the both sides of (3.9), we have

$$
\begin{aligned}
T_{t_{1}}^{(\beta)} F(w) & =-\int_{0}^{\infty} T_{t_{1}}^{(\beta)}\left(\frac{\partial}{\partial t} T_{t}^{(\beta)} f(w)\right) \cdot a(t) d t \\
& =-\int_{0}^{\infty} \frac{\partial}{\partial t} T_{t+t_{1}}^{(\beta)} f(w) \cdot a(t) d t
\end{aligned}
$$

and applying the operator $\sum_{n=0}^{\infty}\left(-n^{\beta}\right) J_{n}$ to the both sides of (3.10), we get

$$
\begin{aligned}
\left|\frac{\partial}{\partial t_{1}} T_{t_{1}}^{(\beta)} F(w)\right| & \leqq \int_{0}^{\infty}\left|\frac{\partial^{2}}{\partial t^{2}} T_{t+t_{1}}^{(\beta)} f(w)\right||a(t)| d t \\
& \leqq K \int_{0}^{\infty}\left|\frac{\partial^{2}}{\partial t^{2}} T_{t+t_{1}}^{(\beta)} f(w)\right| d t \\
& =K \int_{t_{1}}^{\infty}\left|\frac{\partial^{2}}{\partial t^{2}} T_{t}^{(\beta)} f(w)\right| d t
\end{aligned}
$$

where $K=\sup _{t \geq 0}|a(t)|$. Hence

$$
\begin{aligned}
\left(g_{1, \beta}\left(M_{m} f\right)\right)^{2} & =\int_{0}^{\infty} t_{1}\left|\frac{\partial}{\partial t_{1}} T_{t_{1}}^{(\beta)} F(w)\right|^{2} d t_{1} \\
& \leqq K^{2} \int_{0}^{\infty} t_{1}\left(\int_{t_{1}}^{\infty}\left|\frac{\partial^{2}}{\partial t^{2}} T_{t}^{(\beta)} f(w)\right| d t\right)^{2} d t_{1} .
\end{aligned}
$$

Applying the Schwarz inequality,

$$
\begin{aligned}
& \leqq K^{2} \int_{0}^{\infty} t_{1}\left(\int_{t_{1}}^{\infty} t^{2}\left|\frac{\partial^{2}}{\partial t^{2}} T_{t}^{(\beta)} f(w)\right|^{2} d t \frac{1}{t_{1}}\right) d t_{1} \\
& =K^{2} \int_{0}^{\infty}\left(\int_{0}^{t} t^{2}\left|\frac{\partial^{2}}{\partial t^{2}} T_{t}^{(\beta)} f(w)\right|^{2} d t_{1}\right) d t \\
& =K^{2} \int_{0}^{\infty} t^{3}\left|\frac{\partial^{2}}{\partial t^{2}} T_{t}^{(\beta)} f(w)\right|^{2} d t \\
& =K^{2}\left(g_{2, \beta}(f)\right)^{2} .
\end{aligned}
$$

Therefore we have

$$
\left|g_{1, \beta}\left(M_{m} f\right)(w)\right| \leqq K\left|g_{2, \beta}(f)(w)\right|,
$$

and hence we have, by Theorem 3.1, 


$$
\begin{aligned}
\left\|\left(I-J_{0}\right) M_{m} f\right\|_{p} & \leqq\left(a_{p, 1, \beta}\right)^{-1}\left\|g_{1, \beta}\left(M_{m} f\right)\right\|_{p} \\
& \leqq\left(a_{p, 1, \beta}\right)^{-1} K\left\|g_{2, \beta}(f)\right\|_{p} \\
& \leqq \frac{A_{p, 2, \beta}}{a_{p, 1, \beta}} K\|f\|_{p} .
\end{aligned}
$$

On the other hand, $\left(I-J_{0}\right) M_{m} f=M_{m} f$ because $m(0)=0$. Hence we obtain

$$
\left\|M_{m} f\right\|_{p} \leqq A_{p}\|f\|_{p}
$$

where the positive constant $A_{p}$ depends only on $p$.

E. M. Stein showed the $L^{p}$-boundedness of the "Riesz potential" operator $(-L)^{i y}(y \in R)$ by using this theorem. In the same way, using this theorem and the Meyer-Shigekawa theorem (Theorem 2.1), we have the $L^{p}$-boundedness of the "Bessel potential" operator as follows.

THEOREM 3.3. The "Bessel potential" operator $(I-L)^{i y}$ is a bounded operator in $L^{p}(B, \mu)(1<p<\infty)$ for every $y \in R$.

Proof. For every $\lambda>0$ and $y \in R$,

$$
\begin{aligned}
(1+\lambda)^{i y} & =\frac{1+\lambda}{\Gamma(1-i y)} \int_{0}^{\infty} e^{-(\lambda+1) s} s^{-i y} d s \\
& =\frac{1}{\Gamma(1-i y)}\left(1+\frac{1}{\lambda}\right) \cdot \lambda \int_{0}^{\infty} e^{-\lambda s}\left(e^{-s} \cdot s^{-i y}\right) d s .
\end{aligned}
$$

Now we denote

$$
\begin{aligned}
& m_{1}(\lambda)=1+\frac{1}{\lambda}, \\
& m_{2}(\lambda)=\lambda \int_{0}^{\infty} e^{-\lambda s}\left(e^{-s} \cdot s^{-i y}\right) d s .
\end{aligned}
$$

By Theorem 2.1, the multiplier operator

$$
f \longrightarrow \sum_{n=0}^{\infty} m_{1}(n) J_{n} f \quad(f \in \boldsymbol{P})
$$

is $L^{p}$-bounded $(1<p<\infty)$.

On the other hand, since $\left|e^{-s} \cdot s^{-i y}\right| \leqq 1$ for every $s>0$, the multiplier operator

$$
f \longrightarrow \sum_{n=1}^{\infty} m_{2}(n) J_{n} f \quad(f \in \boldsymbol{P})
$$

is also $L^{p}$-bounded $(1<p<\infty)$ by Theorem 3.2. Now we can denote 


$$
(I-L)^{i y} f=J_{0} f+\frac{1}{\Gamma(1-i y)} \sum_{n=1}^{\infty} m_{1}(n) m_{2}(n) J_{n} f
$$

for every $f \in P$. Hence we get

$$
\left\|(I-L)^{i y} f\right\|_{p} \leqq C_{p} \cdot K_{y}\|f\|_{p}
$$

where $C_{p}$ depends only on $p$, and $K_{y}$ depends only on $y$.

REMARK. $K_{y}$ may be regarded as a continuous function of $y \in \boldsymbol{R}$, and

$$
A \leqq K_{y} /\left(\frac{e^{(\pi / 2) y}}{1+|y|}\right) \leqq B
$$

where the positive constants $A, B$ are independent of $y \in \boldsymbol{R}$.

§4. Interpolation theory on Sobolev spaces over the abstract Wiener space.

In this section, we shall prove an interpolation theorem on Sobolev spaces over the abstract Wiener space.

MaIn TheORem. Let $1<p_{0}, p_{1}<\infty$ and $s_{0}, s_{1} \in R$. Then

$$
\left[W^{s_{0}, p_{0}}, W^{s_{1}, p_{1}}\right]_{\theta}=W^{s, p} \quad \text { for every } \theta \in[0,1]
$$

where $s=(1-\theta) s_{0}+\theta s_{1}, 1 / p=(1-\theta) / p_{0}+\theta / p_{1}$. (About these notations, see [9] Appendix B.)

We divide the proof of the theorem into 2 parts, Theorem 4.1 and Theorem 4.2. Without loss of generality, we can assume $s_{0} \leqq s_{1}$.

THEOREM 4.1. Under the same conditions as the Main Theorem,

$$
W^{s, p} \subset\left[W^{s_{0}, p_{0}}, W^{s_{1}, p_{1}}\right]_{\theta} .
$$

In order to prove this theorem, we shall show the following lemmas.

Lemma 4.1. Let $D=\{z \in C \mid 0 \leqq R e . z \leqq 1\}$ and $1 / p_{z}=(1-z) / p_{0}+z / p_{1}$. For every $f \in P$, the function $\Psi$ defined by

$$
\Psi(z)=\left|(I-L)^{s / 2} f\right|^{p / p_{z}}
$$

is an $L^{p_{0}-v a l u e d}$ continuous function on $D$.

Proof. Let $z \in D$ and $z+\Delta z \in D$. Then we have 


$$
\begin{aligned}
\|\Psi(z+\Delta z)-\Psi(z)\|_{p_{0}}^{p_{0}=} & \int_{B}||(I-L)^{g / 2} f(w)^{p / p_{0}+p\left(1 / p_{1}-1 / p_{0}\right)(z+\Delta z)} \\
& -\left.\left|(I-L)^{g / 2} f(w)\right|^{p / p_{0}+p\left(1 / p_{1}-1 / p_{0}\right) z}\right|^{p_{0}} d \mu(w) .
\end{aligned}
$$

Now the integrand is dominated by the function

$$
\Psi_{0}(w)=\left[2 \times \max \left\{1,\left|(I-L)^{s / 2} f\right|\right\}\right]^{q} \quad \text { where } q=\max \left\{p_{0}, p_{1}\right\}
$$

and $\Psi_{0}$ is integrable on $B$. Hence, by the Lebesgue dominated convergence theorem, we have

$$
\lim _{|\Delta z| \rightarrow 0}\|\Psi(z+\Delta z)-\Psi(z)\|_{p_{0}}^{p_{0}}=0
$$

Therefore $\Psi(z)$ is continuous on $D$.

LEMMA 4.2. For every $\varepsilon>0$ and $z \in D$, there exists $\delta>0$ and $f_{\varepsilon, z} \in P$ such that

$$
\left\|\Psi(z+\Delta z)-f_{\varepsilon, z}\right\|_{p_{0}}<\varepsilon \quad \text { for every }|\Delta z|<\delta \text {. }
$$

Proof. Fix $z \in D$. For every $\varepsilon>0$, there exists $\delta>0$ such that

$$
\|\Psi(z+\Delta z)-\Psi(z)\|_{p_{0}}<\varepsilon / 2
$$

for every $\Delta z \in C$ which satisfies $|\Delta z|<\delta$ and $z+\Delta z \in D$. On the other hand, since $\Psi(\cdot) \in L^{p_{0}}$, there exists $f_{\varepsilon, z} \in \boldsymbol{P}$ such that

$$
\left\|\Psi(z)-f_{\varepsilon, z}\right\|_{p_{0}}<\varepsilon / 2
$$

Hence we have proved this lemma.

LEMMA 4.3. Let $s_{z}=(1-z) s_{0}+z s_{1}$. For $f \in P, z \in D$, the function $\Phi$ defined by

$$
\Phi(z)=e^{z^{2}-\theta^{2}}(I-L)^{-z_{z} / 2}\left[\operatorname{sign}\left\{(I-L)^{s / 2} f\right\} \cdot\left|(I-L)^{s / 2} f\right|^{p / p}\right]
$$

is a $W^{s_{0}, p_{0}-v a l u e d}$ continuous function on $D$ where $\operatorname{sign} \zeta=\zeta /|\zeta|$ for every $\zeta \in C$.

Proof. Since $f \in P$ and $s_{0} \leqq s_{1}$, it is easy to show $\Phi(z) \in W^{s_{0}, p_{0}}$ for every $z \in D$. The continuity of $\Phi$ is equivalent to the one of the function

$$
\Phi_{0}(z)=(I-L)^{-z_{z} / 2}\left[\left|(I-L)^{s / 2} f\right|^{p / p_{z}}\right] \text {. }
$$

Now we show the continuity of $\Phi_{0}$. In the following, we denote $\Delta z=$ $\Delta x+i \Delta y$ where $\Delta x, \Delta y \in \boldsymbol{R}$. Fix any $\varepsilon>0$ and let $f_{\varepsilon, z}$ and $\delta>0$ satisfy Lemma 4.2. And take $|\Delta z|<\delta$. Now, 


$$
\begin{aligned}
& \left\|\Phi_{0}(z+\Delta z)-\Phi_{0}(z)\right\|_{s_{0}, p_{0}} \\
& =\left\|(I-L)^{-\left(\boldsymbol{s}_{z}+\Delta z\right) / 2}\left\{\left|(I-L)^{s / 2} f\right|^{p /\left(p_{z}+\Delta z\right)}\right\}-(I-L)^{-\boldsymbol{s}_{z} / 2}\left\{\left|(I-L)^{s / 2} f\right|^{p / p_{z}}\right\}\right\|_{8_{0}, p_{0}} \\
& \leqq\left\|(I-L)^{-\left(\varepsilon_{z}+\Delta z\right) / 2}\left\{\left|(I-L)^{z / 2} f\right|^{p /\left(p_{z}+\Delta z\right)}\right\}-(I-L)^{-\left(\varepsilon_{z}+\Delta_{z}\right) / 2} f_{\varepsilon, z}\right\|_{\varepsilon_{0}, p_{0}} \\
& +\left\|(I-L)^{-\left(s_{z}+\Delta z\right) / 2} f_{\varepsilon, z}-(I-L)^{-z_{z} / 2} f_{\varepsilon, z}\right\|_{\delta_{0}, p_{0}} \\
& +\left\|(I-L)^{-z_{z} / 2} f_{\varepsilon, z}-(I-L)^{-z_{z} / 2}\left\{\left|(I-L)^{s / 2} f\right|^{p /\left(p_{z}+\Delta z\right)}\right\}\right\|_{s_{0}, p_{0}} \\
& +\left\|(I-L)^{s_{z} / 2}\left\{\left|(I-L)^{s / 2} f\right|^{p /\left(p_{z}+\Delta z\right)}\right\}-(I-L)^{-z_{z} / 2}\left\{\left|(I-L)^{s / 2} f\right|^{p / p_{z}}\right\}\right\|_{s_{0}, p_{0}} \\
& =A_{1}+A_{2}+A_{3}+A_{4} \text {, say . }
\end{aligned}
$$

We have

$$
\begin{aligned}
A_{1} & =\left\|(I-L)^{\left(s_{0}-s_{x+\Delta x} / 2\right.}(I-L)^{i(y+\Delta y)\left(s_{0}-s_{1}\right) / 2}\left\{\left|(I-L)^{s / 2} f\right|^{p /\left(p_{z}+\Delta z\right)}-f_{\varepsilon, z}\right\}\right\|^{p_{0}} \\
& \leqq C_{p_{0}} \cdot K_{y+\Delta y} \cdot \varepsilon
\end{aligned}
$$

by Theorem 3.3 and Lemma 4.2. From (3.17), for small $\delta>0$, we can take $K_{y+\Delta y}$ as

$$
K_{y+\Delta y} \leqq 2 K_{y}
$$

Since $f_{\varepsilon, z} \in \boldsymbol{P}, J_{n} f_{\varepsilon, z}=0$ for all but finitely many $n$. Hence we have

$$
\begin{aligned}
A_{2} & =\left\|\left[(I-L)^{-\left(s_{z}+\Delta z\right) / 2}-(I-L)^{-s_{z} / 2}\right] f_{z, \varepsilon}\right\|_{s_{0}, p_{0}} \\
& \leqq \sum_{n=0}^{\infty}\left|(1+n)^{-s_{0} / 2}\left\{(1+n)^{\left(s_{0}-s_{1}\right)(z+\Delta z) / 2}-(1+n)^{\left(s_{0}-s_{1}\right) z / 2}\right\}\right|\left\|J_{n} f_{z, \varepsilon}\right\|_{p_{0}} \\
& <\varepsilon
\end{aligned}
$$

for sufficiently small $\delta>0$. Similarly to the first term, we can get $A_{3}<\varepsilon$, and we have

$$
\begin{aligned}
A_{4} & =\left\|(I-L)^{\left(\varepsilon_{0}-s_{z}\right) / 2}(I-L)^{i y\left(s_{0}-s_{1}\right) / 2}\left\{\left|(I-L)^{s / 2} f\right|^{p /\left(p_{z}+\Delta z\right)}-\left|(I-L)^{s / 2} f\right|^{p / p_{z}}\right\}\right\|_{p_{0}} \\
& \leqq C_{p} \cdot K_{y} \cdot \varepsilon
\end{aligned}
$$

by Theorem 3.3 and Lemma 4.1. Hence $\Phi_{0}$ is continuous.

LEMMA 4.4. For the function $\Phi$ in Lemma 4.3, there exists a constant $M>0$ such that

$$
\|\Phi(z)\|_{s_{0}, p_{0}} \leqq M \quad \text { for every } z \in D
$$

Proof. We have

$$
\begin{aligned}
& \|\Phi(z)\|_{s_{0}, p_{0}} \\
& =\left\|e^{z^{2-\theta^{2}}}(I-L)^{-s_{z} / 2}\left[\operatorname{sign}\left\{(I-L)^{s / 2} f\right\} \cdot\left|(I-L)^{8 / 2} f\right|^{p / p_{z}}\right]\right\|_{8_{0}, p_{0}} \\
& =e^{x^{2}-y^{2}-\theta^{2}}||(I-L)^{\left(s_{0}-s_{z}\right) / 2}(I-L)^{i y\left(s_{0}-s_{1}\right) / 2}\left[\operatorname{sign}\left\{(I-L)^{s / 2} f\right\} \cdot\left|(I-L)^{s / 2} f\right|^{p / p_{z}}\right] \|_{p_{0}}
\end{aligned}
$$




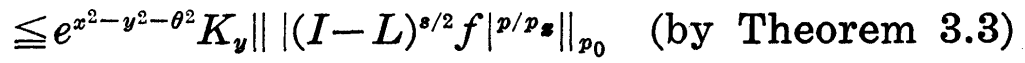

$$
\begin{aligned}
& =e^{x^{2}-y^{2}-\theta^{2}} K_{y}\left[\int_{B}\left|(I-L)^{\varepsilon / 2} f\right|^{p \cdot p_{0} / p_{m}} d \mu\right]^{1 / p_{0}} \\
& \leqq e^{x^{2}-y^{2}-\theta^{2}} K_{y}\left[\int_{B}\left|(I-L)^{s / 2} f\right|^{p \cdot p_{0}} d \mu\right]^{1 / p_{x} \cdot p_{0}} \text {. }
\end{aligned}
$$

Since $K_{y}$ is continuous, and by (3.17), the function

$$
e^{x^{2}-y^{2}-\theta^{2}} K_{y}
$$

is bounded on $D$ (where $z=x+i y$ ). Therefore we get

$$
\begin{aligned}
\|\Phi(z)\|_{8_{0}, p_{0}} & \leqq C\|f\|_{8, p}^{p / p} x_{p_{0}} \\
& \leqq C\left(1+\|f\|_{8, p \cdot p_{0}}\right)=M
\end{aligned}
$$

for every $z \in D$.

LEMMA 4.5. $\Phi$ is a $W^{s_{0}, p_{0}-v a l u e d ~ h o l o m o r p h i c ~ f u n c t i o n ~ o n ~} D_{0}$, the interior of $D$.

Proof. From the general theory of vector-valued holomorphic function (cf. [6]) and Proposition 2.2, it is sufficient to show that the function

$$
F(z)=\int_{B} \Phi(z)(w) g(w) d \mu(w)
$$

is holomorphic on $D_{0}$ for every $g \in W^{-s_{0}, p_{0}^{\prime}}$ where $1 / p_{0}+1 / p_{0}^{\prime}=1$. Now we fix such $g$. Since $\boldsymbol{P}$ is a dense set of $W^{-s_{0}, p_{0}^{\prime}}$, for every $k \in N$, there exists $g_{k} \in P$ such that

$$
\left\|g-g_{k}\right\|_{-s_{0}, p_{0}^{\prime}}<\frac{1}{k}
$$

Then

$$
F(z)=\int_{B} \Phi(z)(w) g_{k}(w) d \mu(w)+\int_{B} \Phi(z)(w)\left[g(w)-g_{k}(w)\right] d \mu(w)
$$

By Lemma 4.4 and Proposition 2.2, we have

$$
\int_{B} \Phi(z)(w)\left[g(w)-g_{k}(w)\right] d \mu(w) \leqq M \times \frac{1}{k} \rightarrow 0 \quad \text { uniformly in } z \text { on } D \text {. }
$$

Hence the sequence of functions $\left\{F_{k}\right\}$;

$$
F_{k}(z)=\int_{B} \Phi(z)(w) g_{k}(w) d \mu(w),
$$


converges to $F(z)$ uniformly on $D$. Therefore, it is sufficient to show the holomorphicity of each $F_{k}(z)$ on $D$. From Lemma 4.3 and the fact $g_{k} \in W^{-s_{0}, p_{0}^{\prime}}$, each $F_{k}(z)$ is continuous on $D$. Now, let $\Gamma$ be any closed curve in $D_{0}$. Then we have

$$
\begin{aligned}
& \int_{\Gamma} F_{k}(z) d z \\
& =\int_{\Gamma} \int_{B} e^{z^{2}-\theta^{2}}(I-L)^{-z_{z} / 2}\left[\operatorname{sign}\left\{(I-L)^{s / 2} f(w)\right\}\right. \\
& \left.\times\left|(I-L)^{s / 2} f(w)\right|^{p / p_{z}}\right] g_{k}(w) d \mu(w) d z \\
& =\int_{\Gamma} \int_{B} e^{z^{2}-\theta^{2}} \operatorname{sign}\left\{(I-L)^{s / 2} f(w)\right\}\left|(I-L)^{s / 2} f(w)\right|^{p / p_{0}+p\left(1 / p_{1}-1 / p_{0}\right) z} \\
& \times(I-L)^{-z_{z} / 2} g_{k}(w) d \mu(w) d z \\
& =\int_{B} \operatorname{sign}\left\{(I-L)^{s / 2} f(w)\right\} \int_{\Gamma} e^{z^{2}-\theta^{2}}\left|(I-L)^{s / 2} f(w)\right|^{p / p_{0}+p\left(1 / p_{1}-1 / p_{0}\right) z} \\
& \times \sum_{n=0}^{\infty}(1+n)^{-s_{0}+z\left(s_{1}-s_{0}\right)} J_{n} f_{k}(w) d z d \mu(w) \\
& =0 \text {. }
\end{aligned}
$$

Here in the last formula of the above estimation, we use the following facts: $J_{n} f_{k}=0$ for all but finite $n$ and $\int_{\Gamma}(\cdot) d z=0$ for other $n$. Hence, by Morera's theorem, each $F_{k}$ is holomorphic on $D_{0}$.

Proof of Theorem 4.1. Fix $f \in \boldsymbol{P}$. Since $W^{s_{0}, p_{0}} \subset W^{s_{0}, p_{0}}+W^{s_{1}, p_{1}}, \Phi$, defined in Lemma 4.3, is continuous on $D$ and holomorphic on $D_{0}$ in the topology of $W^{s_{0}, p_{0}}+W^{s_{1}, p_{1}}$. Similar to Lemma 4.4, we get

$$
\sup _{y}\|\Phi(i y)\|_{s_{0}, p_{0}} \leqq C\|f\|_{s, p}^{p / p_{0}} \text {. }
$$

By Lemma 4.3, $\Phi(i y)$ is a continuous function of $y$. In the same way, we can show

$$
\sup _{y}\|\Phi(1+i y)\|_{s_{1}, p_{1}} \leqq C\|f\|_{s, p}^{p / p_{1}}
$$

and the continuity of $\Phi(1+i y)$ of $y$. Hence we get

$$
f \in\left[W^{s_{0}, p_{0}}, W^{s_{1}, p_{1}}\right]_{\theta},
$$

and we have

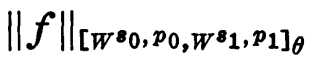

$$
\begin{aligned}
& \leqq \max \left\{\sup \|\Phi(i y)\|_{s_{0}, p_{0}}, \sup _{y}\|\Phi(1+i y)\|_{s_{1}, p_{1}}\right\} \quad \text { (see [8] Appendix B) } \\
& \leqq C\left(\|f\|_{s, p}^{p / p_{0}}+\|f\|_{s, p}^{p / p_{1}}\right) \text {. }
\end{aligned}
$$


Therefore we get

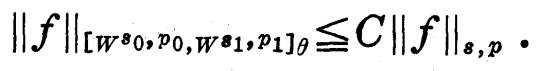

Now we complete the proof.

THEOREM 4.2. Under the same conditions as the Main Theorem,

$$
\left[W^{s_{0}, p_{0}}, W^{s_{1}, p_{1}}\right]_{\theta} \subset W^{s, p} .
$$

Proof. $\quad \boldsymbol{P}$ is a dense subset of $W^{s, p}, W^{s_{0}, p_{0}} \cap W^{s_{1}, p_{1}}$, and $\left[W^{s_{0}, p_{0}}, W^{s_{1}, p_{1}}\right]_{\theta^{\prime}}$. Hence it is sufficient to show that there exists a constant $C^{\prime}>0$ such that

$$
\|f\|_{s, p} \leqq C^{\prime}\|f\|_{\left[W^{\varepsilon_{0}}, p_{0}, W^{\left.\varepsilon_{1}, p_{1}\right] \theta}\right.} \quad \text { for every } f \in \boldsymbol{P} .
$$

Now, let $g \in \boldsymbol{P} \subset W^{-s, p^{\prime}}$ such that $\|g\|_{-s, p^{\prime}} \leqq 1$. From Theorem 4.1 and Proposition 2.2,

$$
W^{-s, p^{\prime}} \subset\left[W^{-s_{0}, p_{0}^{\prime}}, W^{-s_{1}, p_{1}^{\prime}}\right]_{\theta}=\left(\left[W^{s_{0}, p_{0}}, W^{s_{1}, p_{1}}\right]_{\theta}\right)^{*},
$$

hence the functional

$$
f \rightarrow \int_{B} f(w) g(w) d \mu(w)
$$

is a bounded linear functional on $\left[W^{s_{0}, p_{0}}, W^{s_{1}, p_{1}}\right]_{\theta}$. Hence

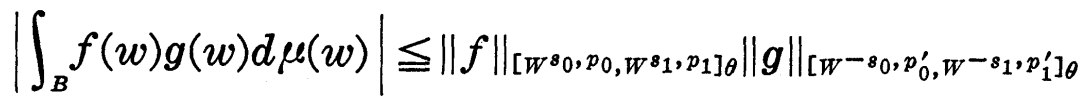

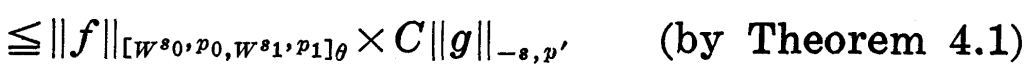

$$
\begin{aligned}
& \leqq C\|f\|_{\left[W^{s_{0}}, p_{\left.0, W^{s_{1}}, p_{1}\right] \theta}\right.} \text {. }
\end{aligned}
$$

Here $C$ is independent of $g$. Therefore we take the supremum with respect to $g$ of both sides at the above inequality and get

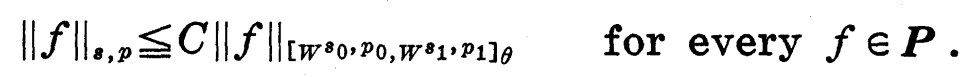

Now we complete the proof.

\section{References}

[1] J. Bergh and J. LöfSTRÖM, Interpolation Spaces, Springer-Verlag, 1976.

[2] R. E. EDWARDS and G. I. GAUDRY, Littlewood-Paley and Multiplier Theory, SpringerVerlag, 1977.

[3] L. Gross, Abstract Wiener spaces, Proceedings of Fifth Berkeley Symp. Math. Statist. Prob. II, part 1 (1965), 31-41, Univ. Calif. Press, Berkeley.

[4] - Logarithmic Sobolev inequalities, Amer. J. Math., 97 (1975), 1061-1083.

[5] T. HIdA, Brownian Motion, Springer-Verlag, 1980. 
[6] N. Nelson, The free Markoff field, J. Funct. Anal., 12 (1973), 211-227.

[7] W. Rudin, Functional Analysis, McGraw-Hill, 1981.

[8] - Real and Complex Analysis, McGraw-Hill, 1986.

[9] C. SADOSKY, Interpolation of Operators and Singular Integrals, Marcel Dekker, 1979.

[10] I. SHIGEKAWA, Derivatives of Wiener functionals and absolute continuity of induced measures, J. Math. Kyoto Univ., 20 (1980), 263-289.

[11] - De Rham-Hodge-Kodaira's decomposition of an abstract Wiener space, J. Math. Kyoto Univ., 26 (1986), 191-202.

[12] E.M. STEIN, Topics in Harmonic Analysis Related to the Littlewood-Paley Theory, Princeton Univ. Press, 1974.

[13] H. Sugita, Sobolev spaces of Wiener functional and Malliavin calculus, J. Math. Kyoto Univ., 25 (1985), 31-48.

[14] - Characterization of Sobolev spaces over an abstract Wiener space, J. Math. Kyoto Univ., 25 (1985), 717-725.

[15] H. TRIEBEL, Interpolation Theory, Function Spaces, Differential Operators, NorthHolland, 1978.

[16] S. Watanabe, Lecture Note of Stochastic Differential Equation and Malliavin Calculus, Tata Inst. Fund. Res., Springer-Verlag, 1984.

Present Address:

Department of Mathematics, Faculty of Science and Technology, Keio University HiYoshi, KoHoKU-KU, YokohaMa 223, JaPAN 